\title{
Complete Genome Sequence Data of Rare Actinomycetes Strain Saccharothrix texasensis 6-C, a Biological Control Agent for Potato Late Blight
}

\author{
Shun Feng, ${ }^{1}$ Yongfei Jian, ${ }^{1}$ Liang Jin, ${ }^{1,2, \dagger}$ Shicai Tang, ${ }^{1}$ and Zhengguo $\mathrm{Li}^{1, \dagger}$ \\ ${ }^{1}$ Key Laboratory of Plant Hormones and Development Regulation of Chongqing, School of Life Sciences, \\ Chongqing University, Chongqing 401331, China \\ ${ }^{2}$ School of Biological Engineering, Chongqing University, Chongqing 401331, China
}

Funding

This research was funded by the National Key R\&D Programme of China, grant number 2017YFE0115500.

\section{Keywords}

biocontrol, genomics, rare actinomycetes, rhizosphere, Saccharothrix sp.

\section{Genome Announcement}

Actinobacteria are widely distributed in both terrestrial and aquatic ecosystems (Barka et al. 2016), and have been major storehouse microorganisms for the discovery of natural products that are highly effective against bacterial, fungal, and oomycete diseases (Choi et al. 2015; De Vrieze et al. 2018; Huang et al. 2007; Zhang et al. 2020). Although many of the discovered natural products have been successfully isolated from the most dominant actinomycetes genus, Streptomyces, novel natural products are increasingly coming from the nonStreptomyces family, known as rare actinomycetes (Choi et al. 2015; Tiwari and Gupta 2012). In actinomycetes, the number of antibiotics or bioactive molecules found thus far corresponds to approximately $10 \%$ or less of potential total number estimated by their biosynthetic genes, meaning that these genes are not expressed under typical laboratory culture conditions (Ding et al. 2019; Tiwari and Gupta 2012; Worsley et al. 2020). Accordingly, genome mining has become a widely practiced alternative way to identify potentially valuable cryptic natural products from actinomycetes. However, relatively few genome mining cases are available in rare actinomycetes, especially in genus Saccharothrix. At present, available records of 4,149 Streptomyces and 36 Saccharothrix genome assemblies can be searched at

\section{†Corresponding authors: L. Jin; jinliang4002@126.com and Z. Li; zgli6@163.com}

Current address for L. Jin and Z. Li: School of Life Sciences, Chongqing University, Chongqing, China.

The author(s) declare no conflict of interest.

Accepted for publication 15 February 2021.

Copyright $\odot 2021$ The Author(s). This is an open access article distributed under the CC BY-NC-ND 4.0 International license. 
Table 1. Statistical table of secondary metabolite synthesis gene clusters ${ }^{a}$

\begin{tabular}{|c|c|c|c|c|}
\hline Cluster ID & Type & Similar cluster & Similarity (\%) & Gene number \\
\hline Cluster 1 & Terpene & Lividomycin biosynthetic gene cluster, saccharide & 6 & 20 \\
\hline Cluster 2 & Lantipeptide & Erythreapeptin biosynthetic gene cluster, RiPP & 75 & 23 \\
\hline Cluster 3 & Terpene & Isorenieratene biosynthetic gene cluster, terpene & 42 & 23 \\
\hline Cluster 4 & Bacteriocin & - & - & 12 \\
\hline Cluster 5 & Terpene & Isorenieratene biosynthetic gene cluster, terpene & 85 & 24 \\
\hline Cluster 6 & T3PKS & Herboxidiene biosynthetic gene cluster, polyketide & 2 & 44 \\
\hline Cluster 7 & Others & - & - & 41 \\
\hline Cluster 8 & NRPS & Erythrochelin biosynthetic gene cluster, NRPS & 85 & 58 \\
\hline Cluster 9 & NRPS-ladderane & Skyllamycin biosynthetic gene cluster, NRPS & 22 & 92 \\
\hline Cluster 10 & Thiopeptide-T2PKS-NRPS & $\begin{array}{l}\text { Sch } 47554 / \text { Sch } 47555 \text { biosynthetic gene cluster, } \\
\text { polyketide }\end{array}$ & 21 & 115 \\
\hline Cluster 11 & T1PKS & Akaeolide biosynthetic gene cluster, polyketide & 32 & 34 \\
\hline Cluster 12 & T1PKS & Meridamycin biosynthetic gene cluster, hybrid & 10 & 41 \\
\hline Cluster 13 & T1PKS-NRPS & Lobosamide biosynthetic gene cluster, T1PKS & 8 & 67 \\
\hline Cluster 14 & Others & Leinamycin biosynthetic gene cluster, hybrid & 4 & 41 \\
\hline Cluster 15 & NRPS & Salinomycin biosynthetic gene cluster, polyketide & 12 & 50 \\
\hline Cluster 16 & Oligosaccharide-others-T1PKS-NRPS & Streptazone E biosynthetic gene cluster, T1PKS & 75 & 116 \\
\hline Cluster 17 & T1PKS & Salinilactam biosynthetic gene cluster, polyketide & 84 & 49 \\
\hline Cluster 18 & T1PKS & Tiacumicin B biosynthetic gene cluster, polyketide & 12 & 29 \\
\hline Cluster 19 & Oligosaccharide & - & - & 62 \\
\hline Cluster 20 & NRPS & Kedarcidin biosynthetic gene cluster, polyketide & 2 & 42 \\
\hline Cluster 21 & Indole & Fortimicin biosynthetic gene cluster, saccharide & 6 & 20 \\
\hline Cluster 22 & Transatpks-T1PKS-NRPS & Teicoplanin biosynthetic gene cluster, NRPS & 9 & 45 \\
\hline Cluster 23 & Terpene & Brasilicardin A biosynthetic gene cluster, terpene & 45 & 24 \\
\hline Cluster 24 & T1PKS & Kedarcidin biosynthetic gene cluster, polyketide & 9 & 42 \\
\hline Cluster 25 & Terpene & SF2575 biosynthetic gene cluster, polyketide & 6 & 16 \\
\hline Cluster 26 & Siderophore & - & - & 17 \\
\hline Cluster 27 & Lantipeptide & Meridamycin biosynthetic gene cluster, hybrid & 13 & 21 \\
\hline Cluster 28 & Others & - & - & 46 \\
\hline Cluster 29 & Lantipeptide & - & - & 21 \\
\hline
\end{tabular}

a Secondary metabolite synthesis gene clusters were predicted by comparing the genome sequences with antiSMASH. NRPS = nonribosomal peptides synthetase and PKS = polyketide synthase.

the GenBank database. Only two complete genomes (assembly levels) of Saccharothrix were reported: Saccharothrix espanaensis (GCA_000328705.1) (Strobel et al. 2012) and S. syringae (GCA_009498035.1) (Mo and Yang 2020). No complete genome of S. texasensis has been reported.

S. texasensis strain 6-C was isolated from the potato rhizosphere and its cell-free fermentation broth has a significant growth inhibitory effect on Phytophthora infestans (unpublished data). Potato late blight induced by $P$. infestans is one of the 10 major oomycete diseases (Kamoun et al. 2015). There are few reports on the inhibition of oomycosis by Saccharothrix spp. Therefore, mining the genome information of $S$. texasensis strain $6-C$ is of great significance for understanding its biocontrol mechanism and discovering new natural products. It might provide new insights for the prevention and control of oomycete diseases.

In this study, genomic DNA of $S$. texasensis strain $6-C$ was extracted using the Bacteria DNA Kit (Omega Bio-Tek Co., Ltd., Guangzhou, China). The genome of $S$. texasensis strain 6-C was sequenced using a combination of Illumina short-read and PacBio long-read sequencing technologies, and sequencing was performed at the Shanghai Majorbio Bio-pharm Technology Co., Ltd. (Shanghai, China). Sequence runs for single-molecule real-time (SMRT) cells were carried out on a PacBio RS II sequencer. SMRT Analysis Portal version 2.1 was used for read filtering and adapter trimming, with default parameters (Cui et al. 2020; Li et al. 2020). Illumina Hiseq $x$-ten read sequences comprising raw reads (>100x) were used to correct base errors of the assembled sequence obtained by the long-read sequences $(>100 x)$. The genome was de novo assembled using the HGAP assembly protocol (Chin et al. 2013). The coding sequence in the genome was predicted by Glimmer, GeneMarkS, and Prodigal software. The transfer RNA (tRNA) and ribosomal RNA (rRNA) were predicted by tRNAscan-SE v2.0 and Barrnap. These databases were used for functional annotation of predicted protein-coding genes, including the gene ontology (GO) (Ashburner et al. 2000), Kyoto Encyclopedia of Genes and Genomes (KEGG) (Aoki-Kinoshita 2006), EuKaryotic 
Orthologous Groups (KOG) (Koonin et al. 2004), GenBank nonredundant (NR) (Koonin et al. 2004), and Swiss-Prot databases (Stutz et al. 2006). In addition, gene clusters involved in secondary metabolite production, antibiotic resistance, toxin synthesis, and carbohydrateactive enzymes (CAZymes) were predicted by comparing the genome sequences with the CAZymes database and antiSMASH (Cui et al. 2020; Yin et al. 2012).

The final assembled genome is a single circular chromosome $(9,045,220 \mathrm{bp})$, without gaps and plasmid, and a GC content of $72.39 \%$. In all, 12 rRNAs and 53 tRNAs were predicted. There were, in total, 8,077 protein-coding genes. Among these genes, 6,390 (79\%) were classified using the KOG database, 4,010 (50\%) were mapped to the Swiss-Prot database, 4,625 (57\%) were assigned GO terms, 1,902 (24\%) were mapped to the KEGG database, and 7,705 (95\%) were mapped to the NR database. Genome analysis using the CAZymes database predicted the existence of 221 glycoside hydrolases, 74 carbohydrate esterases, 69 glycosyl transferases, 20 polysaccharide lyases, 12 carbohydrate-binding modules, and 33 auxiliary activities, which are reported to participate in a diversity of key biological processes such as energy uptake or cell wall degradation (Compain 2020; Gilbert 2010). Genome analysis using antiSMASH predicted the existence of 29 gene clusters involved in secondary metabolite production (Table 1). The top four gene clusters were nonribosomal peptides synthetase (nine clusters), type I polyketide synthase (five clusters), terpene (four clusters), and lanthipeptide (three clusters), and some of the enzymes encoded by such clusters are reported to be involved in the biosynthesis of antimicrobials or antagonistic activity of particular biocontrol bacteria (Li et al. 2014; Malmierca et al. 2013; Repka et al. 2017).

This genome sequence could contribute to the understanding of genetic and genomic diversities of Saccharothrix spp. It could also provide opportunities to analyze the molecular basis of its biocontrol activity. The complete whole-genome sequence of $S$. texasensis strain 6-C has been deposited at GenBank database under the accession number CP064192 (BioProject: PRJNA671555, BioSample: SAMN16534143).

\section{Acknowledgments}

We thank the School of Life Sciences, Chongqing University and its scientific and supporting staff for financial support and management and Zhongkang Wang (Chongqing University) for his guidance and advice on microbiology.

\section{Author-Recommended Internet Resources}

antiSMASH: https://antismash.secondarymetabolites.org/\#!/start Barrnap: https://github.com/tseemann/barrnap

Glimmer: http://ccb.jhu.edu/software/glimmer/index.shtml

tRNAscan-SE v2.0: http://trna.ucsc.edu/software

\section{Literature Cited}

Aoki-Kinoshita, K. F. 2006. Overview of KEGG applications to omics-related research. J. Pestic. Sci. 31:296-299.

Ashburner, M., Ball, C. A., Blake, J. A., Botstein, D., Butler, H., Cherry, J. M., Davis, A. P., Dolinski, K., Dwight, S. S., Eppig, J. T., Harris, M. A., Hill, D. P., Issel-Tarver, L., Kasarskis, A., Lewis, S., Matese, J. C., Richardson, J. E., Ringwald, M., Rubin, G. M., and Sherlock, G. 2000. Gene ontology: Tool for the unification of biology. Nat. Genet. 25:25-29.

Barka, E. A., Vatsa, P., Sanchez, L., Gaveau-Vaillant, N., Jacquard, C., Klenk, H.-P., Clément, C., Ouhdouch, Y., and van Wezel, G. P. 2016. Taxonomy, physiology, and natural products of actinobacteria. Microbiol. Mol. Biol. Rev. 80:1-43.

Chin, C. S., Alexander, D. H., Marks, P., Klammer, A. A., Drake, J., Heiner, C., Clum, A., Copeland, A., Huddleston, J., Eichler, E. E., Turner, S. W., and Korlach, J. 2013. Nonhybrid, finished microbial genome assemblies from long-read SMRT sequencing data. Nat. Methods 10:563-569.

Choi, S. S., Kim, H. J., Lee, H. S., Kim, P., and Kim, E. S. 2015. Genome mining of rare actinomycetes and cryptic pathway awakening. Process Biochem. 50: 1184-1193.
Compain, P. 2020. Multivalent effect in glycosidase inhibition: The end of the beginning. Chem. Rec. 20:10-22.

Cui, Y., Lv, Y., Song, M., Wang, S., Hu, H., Jahan, N., Zhu, B., and Guo, L. 2020. Genome sequence of Micromonospora terminaliae $\mathrm{TMS}^{\top}$, a new endophytic actinobacterium isolated from the medicinal plant Terminalia mucronata. Mol. Plant-Microbe Interact. 33:721-723.

De Vrieze, M., Germanier, F., Vuille, N., and Weisskopf, L. 2018. Combining different potato-associated Pseudomonas strains for improved biocontrol of Phytophthora infestans. Front. Microbiol. 9:2573.

Ding, T., Yang, L. J., Zhang, W. D., and Shen, Y. H. 2019. The secondary metabolites of rare actinomycetes: Chemistry and bioactivity. RSC Adv 9:21964-21988.

Gilbert, H. J. 2010. The biochemistry and structural biology of plant cell wall deconstruction. Plant Physiol. 153:444-455.

Huang, J. W., Shih, H. D., Huang, H. C., and Chung, W. C. 2007. Effects of nutrients on production of fungichromin by Streptomyces padanus PMS-702 and efficacy of control of Phytophthora infestans. Can. J. Plant Pathol. 29:261-267.

Kamoun, S., Furzer, O., Jones, J. D. G., Judelson, H. S., Ali, G. S., Dalio, R. J. D., Roy, S. G., Schena, L., Zambounis, A., Panabières, F., Cahill, D., Ruocco, M., Figueiredo, A., Chen, X. R., Hulvey, J., Stam, R., Lamour, K., Giizen, M., Tyler, B. 
M., Grünwald, N. J., Mukhtar, M. S., Tomé, D F A., Tör, M., Van Den Ackerveken, G., McDowell, J., Daayf, F., Fry, W. E., Lindqvist-Kreuze, H., Meijer, H. J. G., Petre, B., Ristaino, J., Yoshida, K., Birch, P. R. J., and Govers, F. 2015. The Top 10 oomycete pathogens in molecular plant pathology. Mol. Plant Pathol. 16:413-434.

Koonin, E. V., Fedorova, N. D., Jackson, J. D., Jacobs, A. R., Krylov, D. M., Makarova, K. S., Mazumder, R., Mekhedov, S. L., Nikolskaya, A. N., Rao, B. S., Rogozin, I. B., Smirnov, S., Sorokin, A. V., Sverdlov, A. V., Vasudevan, S., Wolf, Y. I., Yin, J. J., and Natale, D. A. 2004. A comprehensive evolutionary classification of proteins encoded in complete eukaryotic genomes. Genome Biol. 5:R7.

Li, Y.-Y., Chen, H.-T., Ding, Y.-J., Xie, Y.-X., Wang, H.-X., Cerny, R. L., Shen, Y.-M., and $\mathrm{Du}$, L.-C. 2014. Iterative assembly of two separate polyketide chains by the same single-module bacterial polyketide synthase in the biosynthesis of antifungal HASF. Angew. Chem. Int. Ed. Engl. 53:7524-7530. https://doi.org/ 10.1002/anie.201403500

Li, Z., Fan, Y., Chang, P., Gao, L., and Wang, X. 2020. Genome sequence resource for Elsinoë ampelina, the causal organism of grapevine anthracnose. Mol. PlantMicrobe Interact. 33:576-579.

Malmierca, M. G., Cardoza, R. E., Alexander, N. J., McCormick, S. P., Collado, I. G., Hermosa, R., Monte, E., and Gutiérrez, S. 2013. Relevance of trichothecenes in fungal physiology: Disruption of tri5 in Trichoderma arundinaceum. Fungal Genet. Biol. 53:22-33.

Mo, X. H., and Yang, S. 2020. Complete genome of nocamycin-producing strain Saccharothrix syringae NRRL B-16468 reveals the biosynthetic potential for secondary metabolites. Curr. Microbiol. 78:107-113.
Repka, L. M., Chekan, J. R., Nair, S. K., and van der Donk, W. A. 2017. Mechanistic understanding of lanthipeptide biosynthetic enzymes. Chem. Rev. 117: 5457-5520.

Strobel, T., Al-Dilaimi, A., Blom, J., Gessner, A., Kalinowski, J., Luzhetska, M., Pühler, A., Szczepanowski, R., Bechthold, A., and Rückert, C. 2012. Complete genome sequence of Saccharothrix espanaensis DSM 44229(T) and comparison to the other completely sequenced Pseudonocardiaceae. BMC Genomics 13:465.

Stutz, A., Bairoch, A., and Estreicher, A. 2006. UniProtKB/Swiss-Prot: The protein sequence knowledgebase. FEBS J. 273:62.

Tiwari, K., and Gupta, R. K. 2012. Rare actinomycetes: A potential storehouse for novel antibiotics. Crit. Rev. Biotechnol. 32:108-132.

Worsley, S. F., Newitt, J., Rassbach, J., Batey, S. F. D., Holmes, N. A., Murrell, J. C., Wilkinson, B., and Hutchings, M. I. 2020. Streptomyces endophytes promote host health and enhance growth across plant species. Appl. Environ. Microbiol. 86:e01053-20.

Yin, Y., Mao, X., Yang, J., Chen, X., Mao, F., and Xu, Y. 2012. dbCAN: A web resource for automated carbohydrate-active enzyme annotation. Nucleic Acids Res. 40:W445-W451.

Zhang, X. Y., Li, C., Hao, J. J., Li, Y. C., Li, D. Z., Zhang, D. M., Xing, X., and Liang, Y. 2020. A novel streptomyces sp. strain PBSH9 for controlling potato common scab caused by streptomyces galilaeus. Plant Dis. 104: 1986-1993. 